\title{
Terahertz multispectral imaging for the analysis of gold nanoparticles' size and the number of unit cells in comparison with other techniques
}

\begin{abstract}
In this work, we investigate the use of a Terahertz multispectral reconstructive imaging technique to measure the size of individual gold nanoparticles and accordingly the number of unit cells in a given gold nanoparticle. The gold nanoparticles were synthesized by the Frens-Turkevich citrate method and characterized by UV-Vis absorption spectroscopy, Dynamic light Scattering, and Transmission Electron Microscopy as well as by terahertz 3D image analysis. The size of the gold nanoparticles as determined by the Terahertz reconstructive imaging was $18.5 \mathrm{~nm}$ which was comparable to the size obtained by the other aforementioned measurement techniques with the exception of Dynamic light scattering which actually measures the hydrodynamic diameter. Using the size of the gold nanoparticles determined by terahertz reconstructive imaging, the number of unit cells in the gold nanoparticle was computed to be 48,825 . The results demonstrate that Terahertz multispectral reconstructive imaging is a powerful nondestructive, non-contact technique for the size analysis of gold nanoparticles.
\end{abstract}

Keywords: nanoparticles, cells, nano-optoelectronic components, bio-photonics, nanosensors, 3D image analysis, nanomaterials
Volume 4 Issue 3 - 2018

\author{
Anis Rahman,' Aunik K Rahman,' William \\ Ghann, ${ }^{2}$ Hyeonggon Kang, ${ }^{2}$ Jamal Uddin ${ }^{2}$ \\ 'Applied Research \& Photonics Inc., Harrisburg, PA, USA \\ ${ }^{2}$ Center for Nanotechnology, Department of Natural Sciences, \\ Coppin State University, Baltimore, MD, USA
}

\begin{abstract}
Correspondence: Jamal Uddin, Center for Nanotechnology, Coppin State University, 2500 W. N. Ave, Baltimore, MD 21216, USA, Tel I-4 I0-95 I-4I I8,Email Juddin@coppin.edu

Anis Rahman, Applied Research \& Photonics Inc., 470 Friendship Road, Suite 10, Harrisburg, PA I7III, USA,
\end{abstract}

Email a.rahman@arphotonics.net

Received: June 12, 2018 | Published: June 25, 2018

\section{Introduction}

Advances in the controlled assembly of nanoscale building blocks, such as gold and silver nanoscale spheres, and quantum dots (QDs) have resulted in functional devices, such as nano-optoelectronic components, bio-photonics, nanosensors, and novel contrast probes for molecular imaging. ${ }^{1,2}$ In the assembled structures, the photophysical properties of nanomaterials are a function of the number and the size of the nanomaterials and the distances among them. For instance, the fluorescence lifetime of a quantum dot cluster depends on the number of the QDs, due to the energy transfer between them. ${ }^{3}$ The surface enhanced Raman spectroscopy (SERS) signal in noble metal nanoparticle arrays depends not only on the properties of the building blocks, but also on the geometric characteristics of the whole array, such as array size. The absorption band of gold nanoparticles is a function of the size and the different fractal structures of the gold nanoparticles and depends on electron-photon relaxation rate. ${ }^{4}$ Therefore, accurate determination of the size parameter of the nanomaterials is important in order to control the photophysical properties of these nanomaterials. Techniques currently deployed in the determination of particle size include Dynamic Light Scattering, ${ }^{5}$ Transmission Electron microscope ${ }^{3}$ Scanning Electron Microscope ${ }^{7}$ and Atomic Force Microscope. ${ }^{8}$ The measured diameter of nanoparticles usually varies depending on the type of instrumentation used for respective measurements. For TEM and SEM, the samples must be small and must remain in high vacuum. Also, for TEM, the samples must be thin enough for electron transparency; as such the sample preparation involves tedious and time consuming steps. AFM imaging is also conducted only on small samples and is strictly a surface imaging technique. In this research, we utilized the terahertz technique as a nondestructive tool capable of non-contact probing and measuring both the size and size distribution of the nanoparticles. Especially, the technique gives one beneficial advantage compared to the AFM,
SEM and TEM. It allows us not only to measure the photophysical properties of materials but also to determine the size dimension of some materials ${ }^{9,10}$ as well as spectroscopic analysis leading to unique identification capabilities.

Terahertz is the region of the electromagnetic spectrum lying between the microwave and the infrared red. ${ }^{11-13}$ Extensive research work has been carried out in the last couple of decades on the generation, detection and application of terahertz within this region. ${ }^{14-19}$ In this paper, Terahertz technique shows promise as a powerful tool for the size analysis of gold nanoparticles and especially as a nondestructive tool without sample size restrictions. Here, particles size of gold nanoparticles has been characterized using terahertz reconstructive imaging technique. ${ }^{20,21}$ Gold nanoparticles exhibit unique properties and have been extensively used in a myriad of applications including, imaging, ${ }^{22-24}$ drug delivery, ${ }^{25-27}$ diagnostics, ${ }^{28,29}$ therapeutics, ${ }^{28}$ sensing ${ }^{30-32}$ and catalysis. ${ }^{33,34}$ In all of these applications, the size of the nanoparticle plays an essential role in the effects they bring to bear. The mean diameter of the gold nanoparticles was also determined using DLS, TEM, FESEM and AFM. Additionally, the number of gold atoms was also calculated from the unit cell consideration. Gold has a face-centered cubic crystal structure; thus there are 4 gold atoms per unit cell (Figure 1). Since gold's lattice parameter is 4.08 $\AA$, its unit cell has a volume of $0.0679 \mathrm{~nm}^{3}$. Even though the unit cells of gold nanocrystals are cubic, gold nanoparticles themselves are pretty much spherical in shape. Knowing the size parameter of a given nanoparticle, the total number of atoms required to constitute each particle may be estimated. In what follows, we describe the results of gold nanoparticles' sizes determined by various competing techniques. Then we demonstrate terahertz reconstructive imaging technique to measure the size of individual gold nanoparticles. We also estimate the number of gold atoms in a nanoparticle from the knowledge of gold crystals structure and the size of the nanoparticle. 


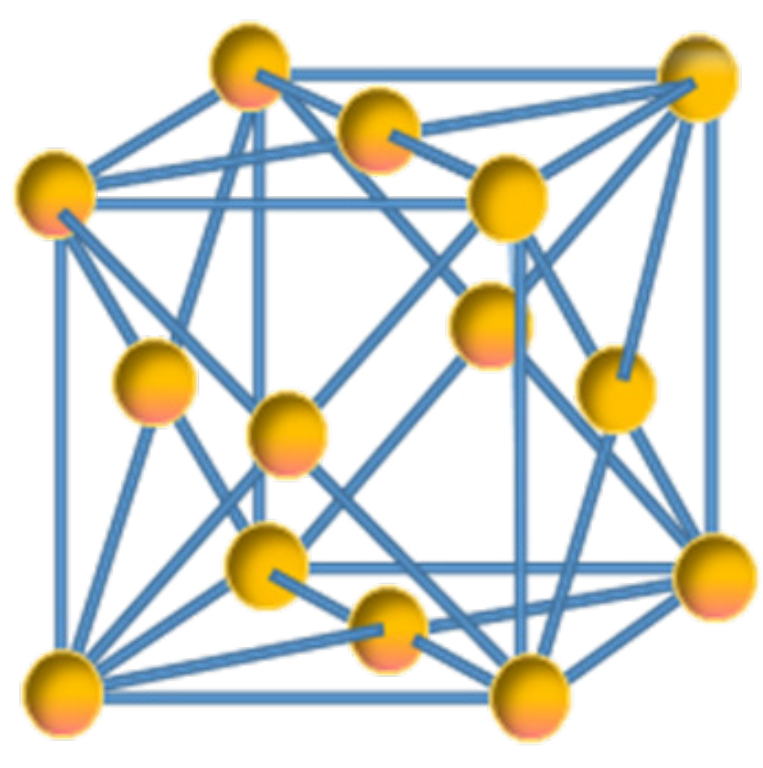

Figure I Unit cell of gold crystal is constituted of 4 gold atoms.

\section{Experimental}

\section{Materials}

All chemicals, reagents and solvents used for this report were of analytical grade and were used without further purification. Tetrachloroauric acid trihydrate $\left(\mathrm{HAuCl}_{4} \cdot 3 \mathrm{H}_{2} \mathrm{O}\right)$ and trisodium citrate $\left(\mathrm{C}_{6} \mathrm{H}_{5} \mathrm{Na}_{3} \mathrm{O}_{7}\right)$ were purchased from Electron Microscopy Services (Fort Washington, PA). The TEM carbon-coated 200 mesh copper grids were purchased from Ted Pella, Inc. (Redding, CA). Doubly purified deionized water from an $18 \mathrm{M}$ Millipore system was used for aqueous solutions. All glassware used for GNPs syntheses was cleaned with freshly prepared aqua regia solution ( 3 parts $\mathrm{HCl}, 1$ part $\left.\mathrm{HNO}_{3}\right)$ and rinsed with Milli-Q water $(\rho>18 \mathrm{M} \Omega)$.

\section{Synthesis of gold nanoparticles}

The gold nanoparticles solution was synthesized using a modified protocol reported elsewhere. ${ }^{35-37}$ The starting materials for the synthesis were gold salt and sodium citrate. A gold salt stock solution was prepared by dissolving $0.1 \mathrm{~g}$ of gold salt in $10 \mathrm{~mL}$ of pure water. Separately, $5 \%$ citrate solution, equivalent to $0.17 \mathrm{M}$, was prepared by dissolving $0.5 \mathrm{~g}$ of sodium citrate in $10 \mathrm{~mL}$ of pure water. Part of the gold salt stock solution was further diluted to obtain $100 \mathrm{~mL}$ of $0.6 \mathrm{mM}$ solution. In a flask equipped with a magnetic stir bar, the gold salt solution was brought to boil with continuous stirring. Next, $3 \mathrm{~mL}$ sodium citrate solution $\left(n_{\text {citrate }} / n_{\mathrm{Au}}=12\right)$ was quickly added to the gold salt solution and the mixture kept under continuous stirring for 20 min. During this period the color of solution changed from light yellow to dark grey, purple, and finally deep ruby red. The reaction was then stopped and the solution was cooled to room temperature. Subsequently, the sizes of the gold nanoparticles in this solution were determined using different techniques described below.

\section{Characterizations}

The size and morphology of the gold nanoparticles (GNPs) were analyzed using Transmission Electron Microscopy (TEM; JEM-1400 Plus) (JEOL USA, Peabody, Massachusetts). The images were viewed using Digital Micrograph software from (Gatan, Inc, Pleasanton, CA). The micrographs were analyzed using Origin software to obtain the mean size of the GNP cores (220 particles were measured) and their associated standard deviations were calculated. The hydrodynamic diameter and size distributions of the GNPs were determined using Dynamic Light Scattering (DLS) (HORIBA, Version LB-550 program). Absorption spectroscopy was carried out with UV-3600 Plus from Shimadzu, MD, USA.

\section{Terahertz measurements}

For terahertz multispectral imaging, the gold nanoparticles were dispersed in a solution. As outlined before, the GNP sample was dispersed in DI water at a concentration of $0.6 \mathrm{mM}$. This solution was further diluted for spin coating on a silicon wafer. That is, $0.58186 \mathrm{~g}$ of the above mentioned GNP solution in DI water was mixed with $0.37031 \mathrm{~g}$ of $\mathrm{MeOH}$ (reagent grade, Sigma-Aldrich). The resulting solution has a concentration of $0.38 \mathrm{mM}$ (Assuming methanol has the same density as water: $1 \mathrm{mg} / \mathrm{mL}$ ). This dilute solution was spun on a prime grade $\mathrm{Si}<100>$ wafer at a casting speed of $2000 \mathrm{rpm}$. A smooth film was obtained. Gold nanoparticles are known to agglomerate in to clusters in DI water. The mixed solvent of methanol-water is expected to disperse the nanoparticles better and help keep them from agglomerating. Therefore, we expect that terahertz imaging will reveal the individual particles rather than their agglomerated clusters.

\section{Results and discussion}

\section{UV-Vis spectroscopy}

The above synthesized gold nanoparticles were first characterized by UV-Vis spectroscopy. Gold nanoparticles are known to exhibit strong absorbance in the visible region with wavelengths within the range of $500 \mathrm{~nm}$ to $600 \mathrm{~nm}$ depending on the size of the particles. It has also been shown that data obtain from UV-Vis measurement could be used to determine the size and concentration of gold nanoparticle..$^{38}$ Figure 2 shows the UV-Vis spectra of the synthesized gold nanoparticles. The wavelength of maximum absorption was $528 \mathrm{~nm}$ consistent of the gold nanoparticles of the size obtained on from DLS. The size of gold nanoparticles was also estimated via UV-Vis spectroscopy according to equations for the computation of size of uncoated spherical gold nanoparticles in water from UVVis spectra. ${ }^{38}$ The equation is based on the ratio of the absorbance of gold nanoparticles at the surface Plasmon resonance peak to the absorbance at $450 \mathrm{~nm}$. Based on this calculation, the mean diameter of the particles was determined to be $17 \mathrm{~nm}$.

\section{Dynamic light scattering}

Dynamic Light Scattering measures the hydrodynamic diameter (HD) or the size of the particles in solution. Particle size obtained using from DLS measurement is usually bigger that those obtained from TEM and FESEM since the electron microscope provides an estimation of the particles in the dry state. Figure 3 shows the DLS measurement of the gold nanoparticles. The average size of gold nanoparticles as determine by DLS was $26.7 \mathrm{~nm}$. The DLS measurement shows only one peak which is indicative of a homogenous or monodispersed gold nanoparticles in solution with negligible agglomeration. However, agglomeration may happen under storing conditions.

\section{Transmission electron microscopy}

The gold nanoparticles were further characterized by transmission 
electron microscopy. To carry out the TEM imaging, a drop of abovementioned aqueous gold nanoparticle solution was dropped on a TEM grid and the sample allowed to dry overnight. Figure 4 shows a representative TEM image of the gold nanoparticles. The average size of the gold nanoparticles calculated from 220 particles was $16.62 \mathrm{~nm}$ with a standard deviation of $\pm 4.73 \mathrm{~nm}$ (Figure 5). However, the overall sizes of the GNPs varied from $\sim 8 \mathrm{~nm}$ to $\sim 34 \mathrm{~nm}$ with a great majority of them lying between $12 \mathrm{~nm}$ and $20 \mathrm{~nm}$. Thus, a small polydispersity is expected. The TEM image also shows that a few GNPs shape is not a perfect sphere; however, an aspect ratio was not established.

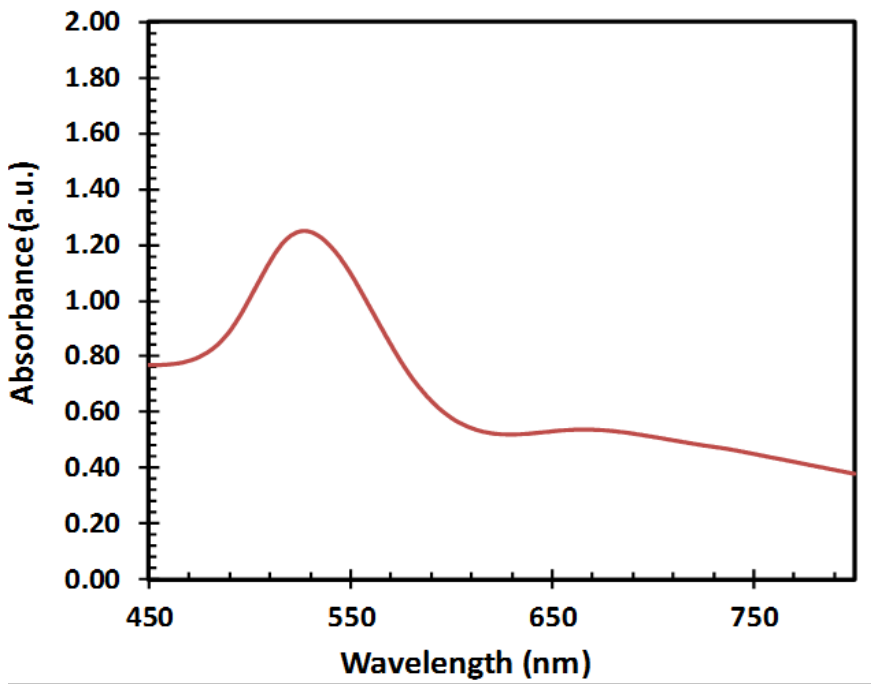

Figure $2 \mathrm{UV}$-Vis spectra of gold nanoparticles.

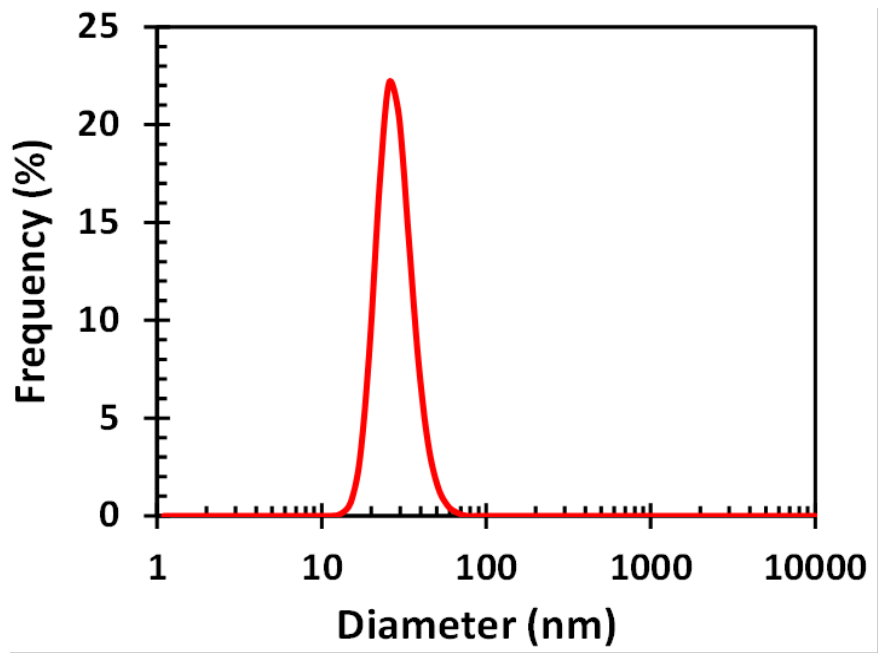

Figure 3 DLS measurement of citrate stabilized gold nanoparticles.

\section{Terahertz reconstructive imaging}

Terahertz measurements were carried out on an integrated terahertz time-domain nanoscanning spectrometer (Applied Research \& Photonics, Harrisburg, PA 17111). A broadband multispectral terahertz source is used for continuous wave terahertz generation via dendrimer dipole excitation mechanism. ${ }^{39}$ The above mentioned Si wafer with the gold nanoparticles was mounted on the nanoscanner. The imaging measurements were done following the procedure outlined in previous reports. ${ }^{20,21}$ Briefly, a reconstructive imaging algorithm was applied on the data obtained by 3D rasterization of 3 $\mu \mathrm{m}^{3}$ volume of the gold nanoparticles on top of the Si wafer. It is assumed that the spin coating process will deposit the majority of the nanoparticles within the $3 \mu \mathrm{m}$ depth of the film on top of the wafer.

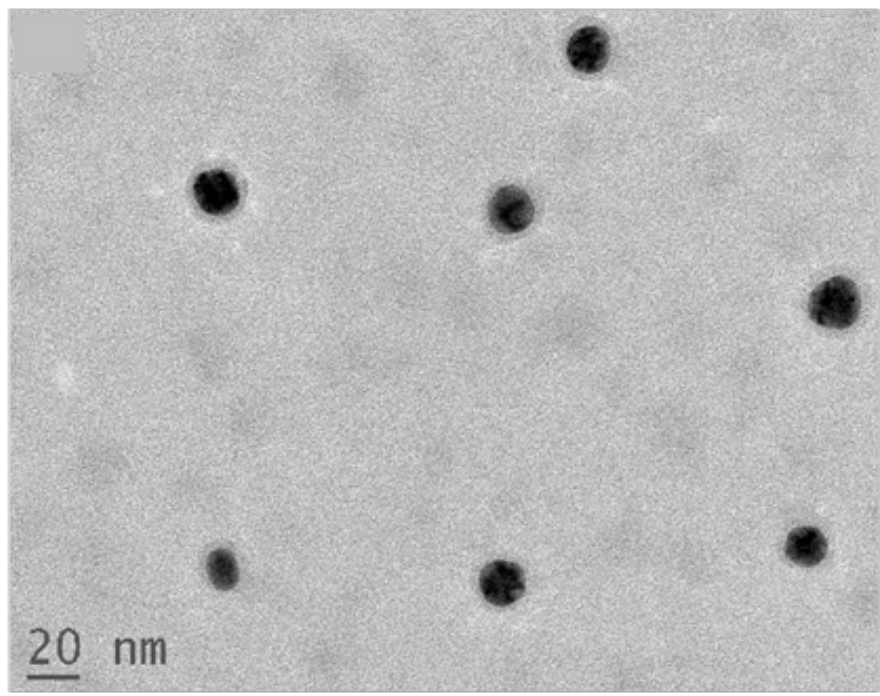

Figure 4 TEM image of the synthesized gold nanoparticles.

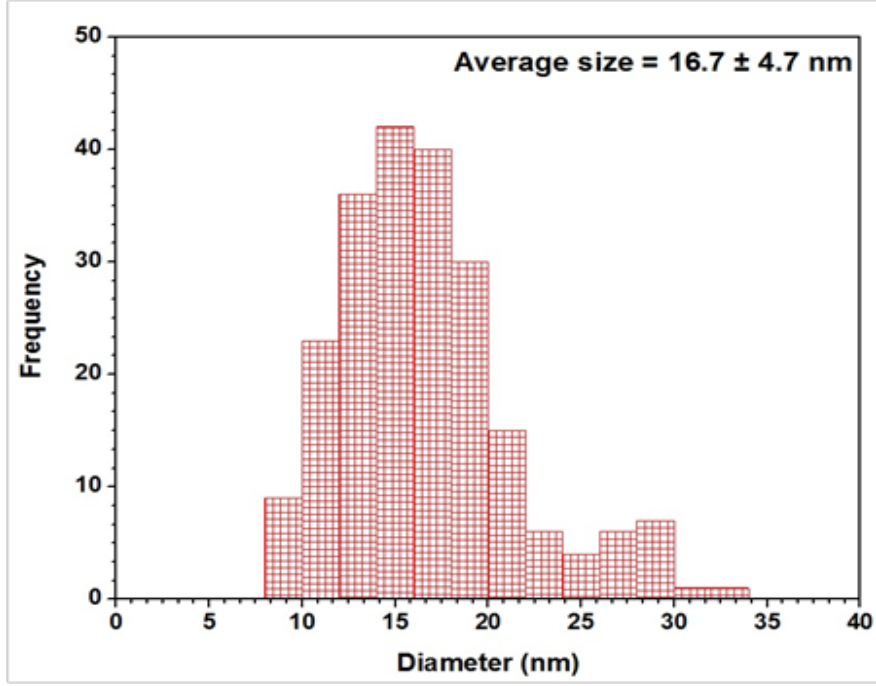

Figure 5 Histogram of gold nanoparticle size distribution.

\section{Terahertz multispectral imaging and particle size analysis}

Terahertz multispectral reconstructive imaging was carried out for size estimate of the gold nanoparticles. Reconstructive imaging details have been described elsewhere. ${ }^{20,39}$ Figure 6 exhibits a surface image of $3 \mu \mathrm{m} \times 3 \mu \mathrm{m}$ area of the aforementioned GNPs spun on $\mathrm{Si}<100>$ wafer. Short range order of the gold nanoparticles is expected from the spin coating process. Measurement was conducted on a layer by layer basis over 3 cubic micron volume. Figure 7 displays a 3D image of 200 cubic nanometer volume extracted from Figure 6. Similarly, Figure 8 shows the top surface of Figure $7 \&$ Figure 9 shows a close up of two gold nanoparticles extracted from Figure 8. A graphical analysis of a nanoparticle from Figure 9 is shown in Figure 10 along the yellow line. This particle is $\sim 18.5 \mathrm{~nm}$ in diameter 
with some fine structure visible in Figure 9 and also in the profile plot of Figure 10. In view of Figure 8, it seems reasonable to assume that not all particles are exactly of the same size, thus indicative of slight polydispersity. However, since Figure 8 is a single plane across the 3D image and not all GNPs are on the same plane, hence, some
GNPs may appear of different size. But as was evident from the TEM image analysis, it is reasonable to assume that the present GNPs may possess light polydispersity. A summary of the gold nanoparticles' sizes obtained via different techniques and their respective atomic constituents is enumerated in Table 1.

Table I Comparison of gold nanoparticles size obtained from various techniques

\begin{tabular}{llll}
\hline Technique & Size $(\mathbf{n m})$ & $\begin{array}{l}\text { Number of unit cells/ } \\
\text { atoms }\end{array}$ & Comments \\
\hline TEM & 16.62 & $35401.5 / 141606$ & Size of dry GNPs on a copper grid \\
DLS & 26.7 & - & Hydrodynamic diameter of GNPs in solution \\
UV-VIS & 17.0 & $37885.7 / 151543$ & Via surface Plasmon resonance \\
Terahertz & 18.5 & $48825.2 / 195301$ & Terahertz multispectral reconstructive imaging \\
\hline
\end{tabular}

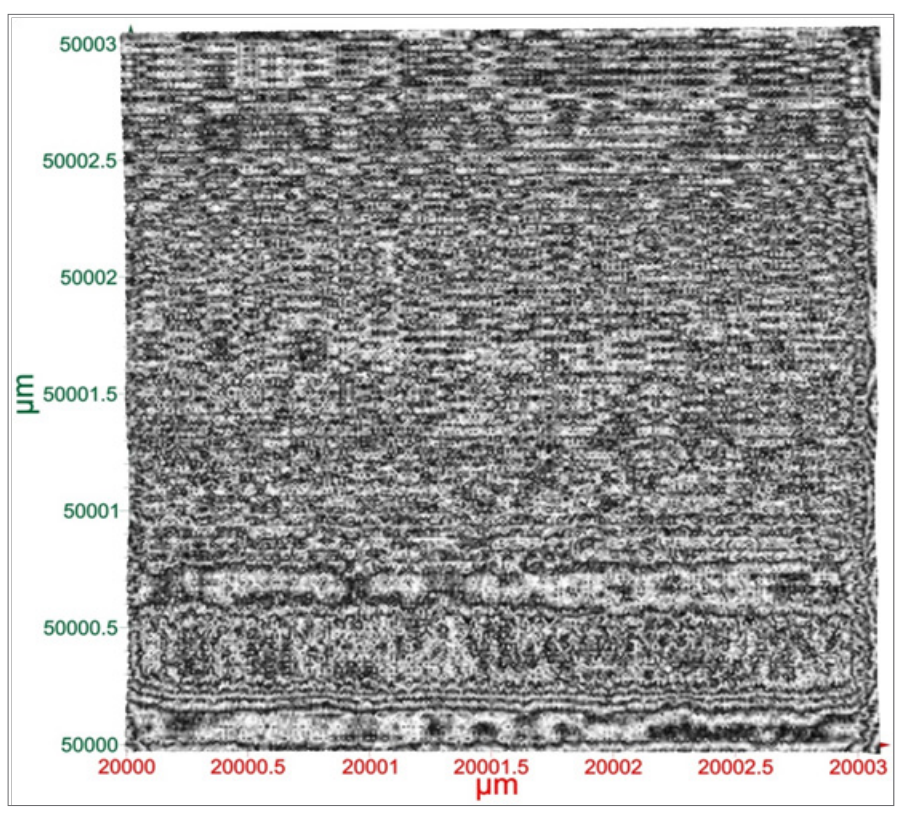

Figure 6 Surface image of $3 \mu \mathrm{m} \times 3 \mu \mathrm{m}$ area of gold nanoparticles spun on $\mathrm{Si}$ wafer. Short range order of the gold nanoparticles is expected from the spin coating process. Measurement was conducted on 3 cubic micron volume.

\section{Calculation of number of atoms}

It is apparent that the gold nanoparticles themselves are pretty much spherical in shape. Since the estimated diameter a gold nanoparticle of the present investigation is $18.5 \mathrm{~nm}$, total volume of the nanoparticle is $\frac{4}{3} \pi r^{3}=3315.23 \mathrm{~nm}^{3}$. Since the unit cell is a face centered cube with length parameter $0.408 \mathrm{~nm}$, this yields the unit cell volume of 0.0679 cubic $\mathrm{nm}$ and thus, the number of unit cell in the nanoparticle $=48825.2$. Thus the total number of gold atoms within this particular nanoparticle is $\sim 195301$.

\section{Conclusion}

Gold nanoparticles were synthesized via the Frens-Turkevich method. These particles were characterized by UV-Vis, DLS, TEM, and also by terahertz 3Dmultispectral reconstructive imaging. It was found that the measured size varies between different techniques indicating some disagreements among various techniques. It is assumed that the variations in the particles measured sizes obtained using, UV-Vis, DLS, and TEM, is resulted from the variations of different parameters employed in the determination of particles size using those techniques. Especially, the terahertz reconstructive 3D imaging technique is capable of determining the size of individual gold nanoparticles more accurately compared to the UV-Vis and DLS. While the average size of the GNPs from TEM analysis was estimated to be $(16.62 \pm 4.73) \mathrm{nm}$, the terahertz technique yielded a size of $18.5 \mathrm{~nm}$. Thus these two instruments produced results that are close to what was obtained from the UV-Vis spectroscopy (viz., $17.0 \mathrm{~nm}$ ). The terahertz imaging technique also gives us more benefits as a nondestructive, non-contact route with sub-surface imaging capabilities. Taking advantage of the FCC crystal structure of gold, the number of unit cells in each gold nanoparticle was computed, from which the number of gold atoms in each nanoparticle was estimated. This feature is useful for differentiating between different routes for GNP synthesis. The technique may also be deployed for other types of nanomaterials.

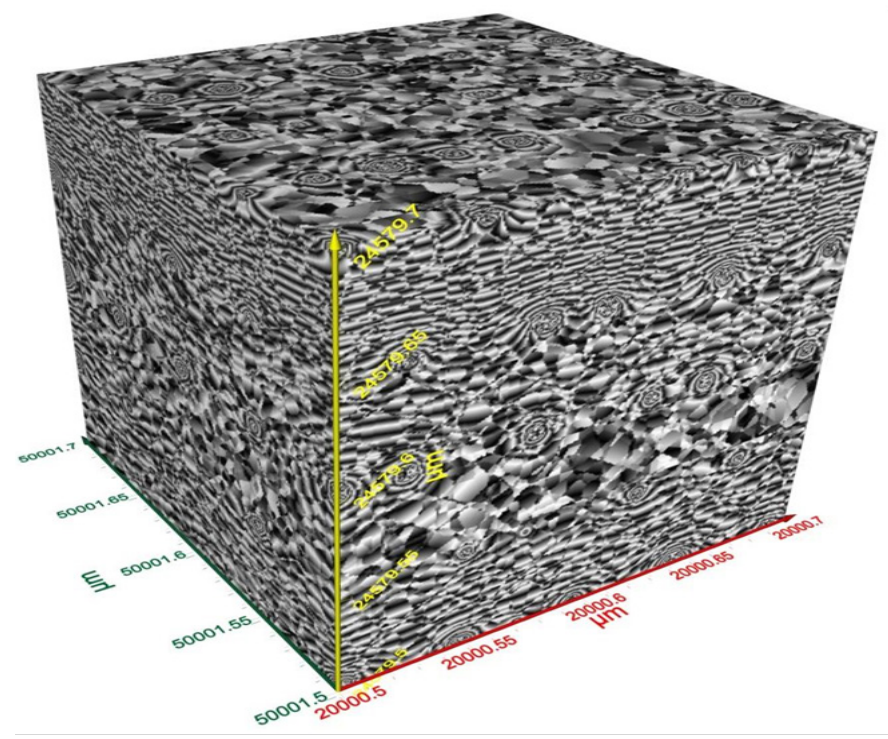

Figure 7 Two hundred (200) cubic nanometer volume extracted from Figure 6. Nanoparticles are seen on the surface as well as scattered in the volume. 


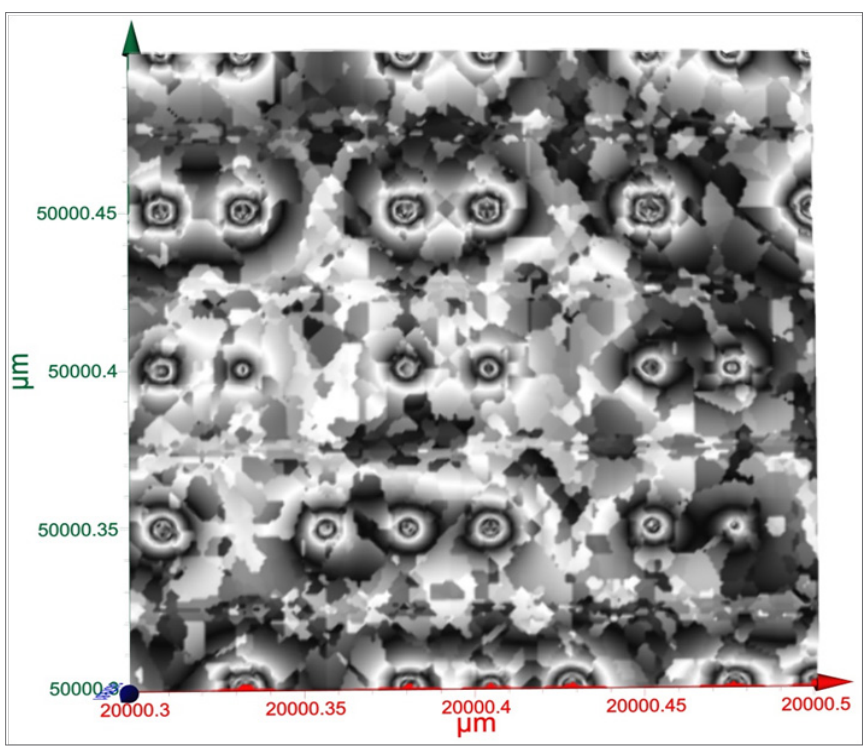

Figure $8 \mathrm{~A}$ surface area of two hundred nanometer square extracted form Figure 6. Close up of two gold nanoparticles from Fig. 5.

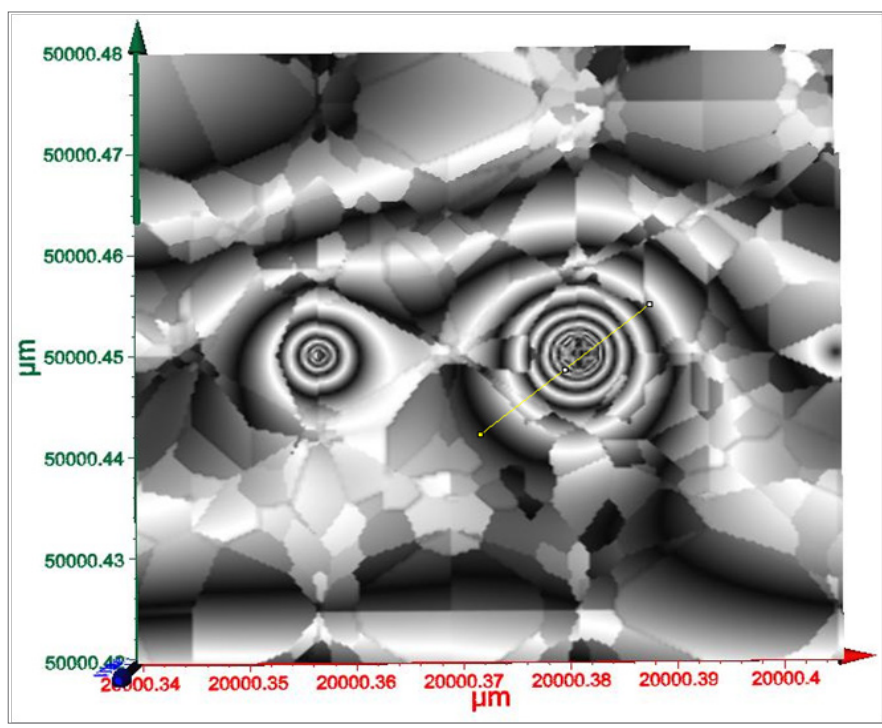

Figure 9 Close up of two gold nanoparticles from Figure 8.

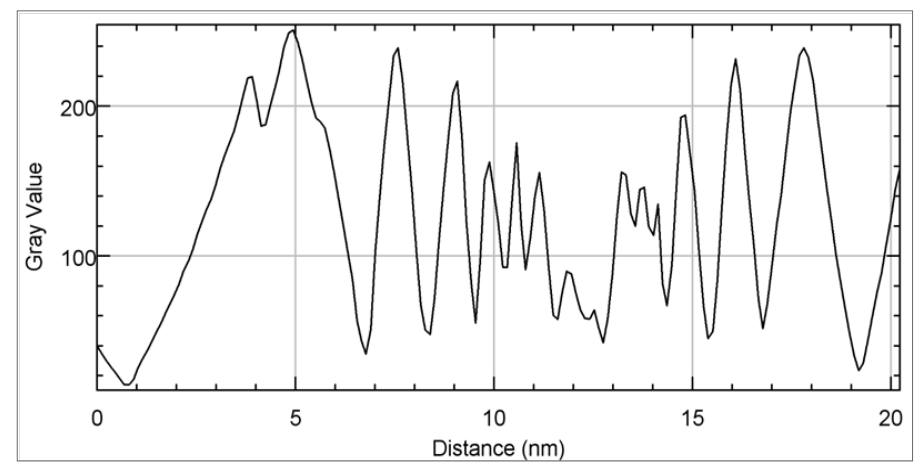

Figure 10 The nanoparticle on the right of Figure 9 has a diameter $\sim 18.5 \mathrm{~nm}$. The fine structure within the particle is visible and may be quantified from Figure 10.

\section{Acknowledgments}

This work was partially supported by the University of Maryland System through Wilson E. Elkins Professorship grant (2485897), Constellation, an Exelon Company through E2- Energy to Educate grant program (163893), and Dept. of Education through SAFRA Title III Grant (2486005). The authors are also grateful to the Institution of Advancement, Coppin State University, for administrative help. The terahertz multispectral imaging was conducted at and supported by Applied Research \& Photonics, Harrisburg, PA. The content is exclusively the responsibility of the authors and does not necessarily represent the official views of the funding agencies.

\section{Conflict of interest}

Author declares that there is no conflict of interest.

\section{References}

1. Adams SM, Campione S, Capolino F. Directing Cluster Formation of Au Nanoparticles from Colloidal Solution. Langmuir. 2013;29:4242-4251.

2. Kang H, Clarke ML, Tang J, et al. Multimodal, Nanoscale, Hyperspectral Imaging Demonstrated on Heterostructures of Quantum Dots and DNAWrapped Single-Wall Carbon Nanotubes. ACS Nano. 2009;3(11):37693775 .

3. Kang H, Clarke ML, Lacerda SH, et al. Multimodal optical studies of single and clustered colloidal quantum dots for the long-term optical property evaluation of quantum dot-based molecular imaging phantoms. Biomedical Optics Express. 2012;3(6):1312-1325.

4. Eustis S, El-Sayed MA. Why gold nanoparticles are more precious than pretty gold: Noble metal surface plasmon resonance and its enhancement of the radiative and nonradiative properties of nanocrystals of different shapes. Chem Soc Rev. 2006;35(3):209-217.

5. Nobbmann U, Connah M, Fish B, et al. Dynamic light scattering as a relative tool for assessing the molecular integrity and stability of monoclonal antibodies. Biotechnol Genet Eng Rev. 2007;24:28.

6. Murphy CJ, Gole AM, Stone JW, et al. Gold Nanoparticles in Biology: Beyond Toxicity to Cellular Imaging. Acc Chem Res. 2008;14(12):17211730 .

7. Liu L, Li B, Ying J, et al. Synthesis and characterization of a new trifunctional magnetic-photoluminescent-oxygen-sensing nanomaterial. Nanotechnology. 2008;19(49):495709.

8. Shukla R, Bansal V, Chaudhary M, et al. Biocompatibility of Gold Nanoparticles and Their Endocytotic Fate Inside the Cellular Compartment: A Microscopic Overview. Langmuir. 2005;21(23):1064410654 .

9. Valverde-Chavez DA, Carlito S Ponseca, Constantinos C. Stoumpos, et al. Intrinsic femtosecond charge generation dynamics in single crystal CH3NH3PbI3. Energy \& Environmental Science. 2015;8(12):37003707.

10. Palka N, Soufiene Krimi, Frank Ospald, et al. Precise Determination of Thicknesses of Multilayer Polyethylene Composite Materials by Terahertz Time-Domain Spectroscopy. Journal of Infrared, Millimeter, and Terahertz Waves. 2015;36(6):578-596, 2015.

11. Beard MC, Turner GM, Schmuttenmaer CA. Terahertz Spectroscopy. The Journal of Physical Chemistry B. 2002;106(29):7146-7159.

12. McIntosh AI, Yang B, Goldup SM, et al. Terahertz spectroscopy: a powerful new tool for the chemical sciences? Chemical Society Reviews. 2012;41(6):2072-2082. 
13. Tonouchi M. Cutting-edge terahertz technology. Nat Photon 2007;1(2):97-105.

14. Ashworth PC, Emma Pickwell-Mac Pherson, Elena Provenzano, et al Terahertz pulsed spectroscopy of freshly excised human breast cancer. Optics Express. 2009;17(15):12444-12454.

15. Kulesa C. Terahertz Spectroscopy for Astronomy: From Comets to Cosmology. IEEE Transactions on Terahertz Science and Technology. 2011;1(1):232-240.

16. Liu HB, Zhong H, Karpowicz N, et al. Terahertz Spectroscopy and Imaging for Defense and Security Applications. Proceedings of the IEEE. 2007;95(8):1514-1527.

17. Rahman A. Dendrimer based terahertz time-domain spectroscopy and applications in molecular characterization. Journal of Molecular Structure. 2011;1006(1-3):59-65.

18. Rahman A, Frenchek S, Kilfoyle B, et al. Diffision Kinetics \& Permeation Concentration of Human Stratum Corneum Characterization by Terahertz Scanning Reflectometry. Drug Development and Delivery. 2012;12(4):43-49.

19. Ghann W, Rahman A, Uddin J. Interaction of Sensitizing Dyes with Nanostructured $\mathrm{TiO}_{2}$ Film in Dye-Sensitized Solar Cells Using Terahertz Spectroscopy. Scientific Reports. 2016;6:30140.

20. Rahman An, Rahman Au ,Yamamoto T. Terahertz Sub-Nanometer SubSurface Imaging of 2D Materials. J Biosens Bioelectron. 2016;7(3):1-8.

21. Rahman An, Rahman Au. Terahertz Spectroscopic Analysis and Multispectral Imaging of Epitaxially Grown Semiconductors with Nanometer Resolution. J Biosens Bioelectron. 2016;7(4):1-6.

22. Ghann WE, Young-Seung $\mathrm{Kim}, \mathrm{Su} \mathrm{Xu}$, et al. Bifunctional gold nanoparticles for targeted dual imaging of angiotensin converting enzyme. SPIE. 2013; 8719:87190U-87190U-9.

23. Ghann WE, Aras O, Fleiter T, et al. Synthesis and Biological Studies of Highly Concentrated Lisinopril-Capped Gold Nanoparticles for CT Tracking of Angiotensin Converting Enzyme (ACE). SPIE. $2011 ; 80250 \mathrm{H}-12$.

24. Hainfeld JF, Slatkin DN, Focella TM, et al. Gold nanoparticles: a new X-ray contrast agent. Br J Radiol. 2006;79(939):248-53.

25. Daniel MC, Margaret E Grow, Hongmu Pan, et al. Gold nanoparticlecored poly(propyleneimine) dendrimers as a new platform for multifunctional drug delivery systems. New Journal of Chemistry. 2011;35(10):2366-2374.

26. Paciotti GF, Kingston DGI, Tamarkin L. Colloidal gold nanoparticles: a novel nanoparticle platform for developing multifunctional tumortargeted drug delivery vectors. Drug Dev Res. 2006;67(1):47-54.
27. Cheng Y, C Samia A, Meyers JD, et al. Highly Efficient Drug Delivery with Gold Nanoparticle Vectors for in Vivo Photodynamic Therapy of Cancer. J Am Chem Soc. 2008;130(32):10643-7.

28. Boisselier E, Astruc D. Gold nanoparticles in nanomedicine: preparations, imaging, diagnostics, therapies and toxicity. Chem Soc Rev. 2009;38(6):1759-82.

29. I El-Sayed IH, Huang X, El-Sayed MA. Surface Plasmon Resonance Scattering and Absorption of anti-EGFR Antibody Conjugated Gold Nanoparticles in Cancer Diagnostics: Applications in Oral Cancer. Nano Lett. 2005;5(5):829-34.

30. Saha K, Agasti SS, Kim C, et al. Gold Nanoparticles in Chemical and Biological Sensing. Chem Rev. 2012;112(5):2739-79.

31. Wang Z, Ma L. Gold nanoparticle probes. Coordination Chemistry Reviews. 2009;253(11-12):1607-1618.

32. Sperling RA, Rivera Gil P, Zhang F, et al. Biological applications of gold nanoparticles. Chemical Society Reviews. 2008;37(9):1896-1908.

33. Bhargava SK, Booth JM, Agrawal S. Gold Nanoparticle Formation during Bromoaurate Reduction by Amino Acids. Langmuir. 2005;21(13):59495956.

34. Daniel MC, Astruc D. Gold Nanoparticles: Assembly, Supramolecular Chemistry, Quantum-Size-Related Properties, and Applications toward Biology, Catalysis, and Nanotechnology. Chem Rev. 2004;104(1):293346.

35. Frens G. Controlled nucleation for regulation of particle-size in monodisperse gold suspensions. Nat Phys Sci. 1973;241:848.

36. Zabetakis K, Ghann W, Kumar S. Effect of high gold salt concentrations on the size and polydispersity of gold nanoparticles prepared by an extended Turkevichâ€Frens method. Gold Bulletin. 2012;45(4):203-211.

37. Ghann WE, Aras O, Fleiter T, et al. Syntheses and Characterization of Lisinopril-Coated Gold Nanoparticles as Highly Stable Targeted CT Contrast Agents in Cardiovascular Diseases. Langmuir. 2012;28(28):10398-10408.

38. Haiss W, Thanh NTK, Aveyard J. Determination of Size and Concentration of Gold Nanoparticles from UV-Vis Spectra. Anal Chem. 2007;79(11):14215-4221.

39. Rahman An, Rahman Au, Tomalia D. Dendrimer Dipole Excitation:A New Mechanism for Terahertz Generation. J Biosens Bioelectron. 2016;7(196):1-6. 